with a reduction to $24 \pm 13(\mathrm{p}<0.01)$ by TP 3 . Myocardial segments with oedema also had increased signal on LGE at TP1 $(\kappa=0.77$; $\mathrm{p}<0.001$ ). At TP1, the proportion of segments with wall motion impairment increased in relation to the extent of both myocardial oedema $(p<0.01)$ and LGE $(p<0.01)$. The volume of LGE decreased significantly between TP1 and TP4 ( $27 \pm 15 \%$ vs $22 \pm 12 \%$; $=0.002)$. Of segments showing LGE at $48 \mathrm{~h}, 50 \%$ showed resolution by 6 months. In segments with such a reduction in LGE, $65 \%$ also showed improved wall motion $(p<0.0001)$. The area of LGE measured at 6 months correlated more strongly with 48-h troponin $(\mathrm{R} 2=0.84 ; \mathrm{p}<0.01)$ than at TP1 $(\mathrm{R} 2=0.5)$. The difference in LGE between TP1 and TP4 had profound effects on the calculation of salvage index $(26 \pm 21 \%$ at TP1 vs $42 \pm 23 \%$ at TP4; $<<0.02)$.

Conclusions (1) Myocardial oedema was unchanged over the first week but decreased by 15 days; (2) a large majority of segments that were positive for oedema also showed LGE, assessed at 12-48 h; (3) In $46 \%$ of patients, LGE present on early scans had diminished in size by 6 months, (4) resolution of LGE was associated with improvement in function; (5) the reduction in LGE at the later time had a profound effect on the calculation of salvage index, which varied by up to $\sim 60 \%$, depending on the time point used. (6) From a clinical perspective, the use of acute LGE may severely underestimate salvaged myocardium and should not be used to predict recovery of myocardial function.

\section{INVESTIGATION OF IL-1 INHIBITION IN PATIENTS PRESENTING WITH NON-ST ELEVATION MYOCARDIAL INFARCTION ACUTE CORONARY SYNDROMES (THE MRC ILA HEART STUDY)}

doi:10.1136/heartjnl-2011-300198.15

\begin{abstract}
${ }^{1} \mathrm{~A}$ C Morton, ${ }^{2} \mathrm{C}$ Foley, ${ }^{1} \mathrm{~A}$ Rothman, ${ }^{1} \mathrm{~J}$ Gunn, ${ }^{3} \mathrm{~J} \mathrm{P}$ Greenwood, ${ }^{3} \mathrm{~A}$ Hall, ${ }^{4} \mathrm{~K}$ Fox, ${ }^{2} \mathrm{~B}$ Lees, ${ }^{2} \mathrm{M}$ Flather, ${ }^{1} \mathrm{D}$ Crossman. ${ }^{1} \mathrm{~N} H \mathrm{H}$ Cardiovascular Biomedical Research Unit, Sheffield, UK; ${ }^{2}$ CTEU, Royal Brompton and Harefields NHS Trust, London, UK; ${ }^{3}$ Academic Unit of Cardiovascular Medicine, Leeds, UK; ${ }^{4}$ Royal Infirmary of Edinburgh, Edinburgh, UK
\end{abstract}

Background Inflammatory mechanisms are involved in both coronary atherogenesis and its presentation as acute coronary syndromes (ACS). To date, drugs used at the time of ACS, or for primary and secondary prevention have not primarily targeted inflammatory mechanisms. Studies with aspirin and statin drugs indicate that anti-inflammatory properties of these compounds may contribute to their beneficial effects. Pre-clinical studies from our group have indicated that the pro-inflammatory cytokine IL-1 drives a number of vascular events relevant to coronary artery disease and ACS. IL-1 can be inhibited by IL-1 receptor antagonist (IL-1ra, Anakinra, Amgen) which is licensed for the treatment of rheumatoid arthritis.

Aims To investigate the effects of interleukin-1 receptor antagonist (IL-1ra) on inflammatory biomarkers in patients with ACS $<48 \mathrm{~h}$ from symptom onset, and to evaluate the safety and tolerability of treatment.

Design and methods The UK MRC ILA-HEART study is an investigator-initiated, non-industry sponsored, phase 2, multi-centre, placebo-controlled trial, comparing the IL-1ra (100 mg) with matching placebo given as a single, daily subcutaneous injection over 2 weeks. The primary outcome of the study was area under the curve (AUC) of high sensitivity CRP (hs-CRP) over the first 7 days of treatment, and the main secondary outcomes are AUC of troponin and safety and compliance of trial treatment. Patients were encouraged to self-administer trial treatment, and underwent daily assessment of hs-CRP, troponin, von Willebrand factor and other biomarkers up to 7 days, and again at 2 weeks and 30 days. Patients were followed up to 12 months for safety (Abstract 15 table 1).
Abstract 15 Table 1 Log transformed values for the primary and secondary outcomes

\begin{tabular}{llclll}
\hline Variable & $\begin{array}{l}\text { Active } \\
\mathbf{n}\end{array}$ & $\begin{array}{l}\text { Active } \\
\text { mean (SD) }\end{array}$ & $\begin{array}{l}\text { Placebo } \\
\mathbf{n}\end{array}$ & $\begin{array}{l}\text { Placebo } \\
\text { mean (SD) }\end{array}$ & $\mathbf{p}$ \\
\hline hsCRP AUC (days 1-7) & 82 & $3.51(1.42)$ & 78 & $3.55(1.46)$ & 0.86 \\
hsCRP at day 7 & 78 & $1.03(1.46)$ & 78 & $1.09(1.91)$ & 0.83 \\
hsCRP at day 14 & 77 & $1.14(1.54)$ & 76 & $0.89(1.56)$ & 0.32 \\
hsCRP at day 30 & 76 & $1.13(0.32)$ & 76 & $0.92(1.15)$ & 0.30 \\
& & & & & \\
Troponin AUC (days 1-7) & 82 & $1.35(1.88)$ & 78 & $1.50(1.96)$ & 0.61 \\
Troponin at day 14 & 77 & $-3.73(2.31)$ & 76 & $-4.14(2.35)$ & 0.27 \\
Troponin at day 30 & 76 & $-4.83(2.44)$ & 76 & $-4.74(2.51)$ & 0.82 \\
& & & & & \\
vWF AUC (days 1-3) & 89 & $1.33(0.35)$ & 84 & $1.31(0.35)$ & 0.66 \\
vWF at day 14 & 77 & $0.36(0.35)$ & 84 & $0.28(0.41)$ & 0.23 \\
vWF at day 30 & 78 & $0.37(0.34)$ & 75 & $0.29(0.43)$ & 0.19 \\
& & & & & \\
IL-6 AUC (days 1-3) & 86 & $2.62(1.13)$ & 83 & $2.56(1.02)$ & 0.74 \\
IL-6 at day 14 & 77 & $1.32(1.35)$ & 77 & $0.92(0.75)$ & 0.028 \\
IL-6 at day 30 & 73 & $1.22(0.92)$ & 75 & $1.08(0.77)$ & 0.31 \\
\hline
\end{tabular}

Results Five UK centres randomised 182 patients with non-ST elevation (NSTEMI) ACS to IL-1ra or placebo. Enrolment completed in March 2010. Mean age was 61 years, 32\% female, 28\% prior MI, $15 \%$ diabetes, $90 \%$ were receiving a statin at the time of randomisation and $64 \%$ had early PCI or CABG. Compliance was good with $85 \%$ of patients receiving daily injections during the first 7 days, and $70 \%$ of patients were able to self-administer the injections. Injection site reactions reported as adverse events occurred in $14 \%$ of patients. There was no significant difference in area under the curve for hsCRP between active and placebo groups. The MACE and serious adverse event rates are shown in Abstract 15 table 2.

\section{Abstract 15 Table 2 MACE and other SAE}

\begin{tabular}{llll}
\hline Event & Active (n $\mathbf{( 9 5 \% ~ C l ) )}$ & Placebo (n $\mathbf{9 5 \%} \mathbf{~ C I}))$ & $\mathbf{p}$ \\
\hline MACE at 30 days & $6(0.19$ to 1.79$)$ & $10(1)$ & 0.35 \\
MACE at 3 months & $10(0.32$ to 1.84$)$ & $13(1)$ & 0.54 \\
MACE at 1 year & $20(0.59$ to 2.22) & $18(1)$ & 0.69 \\
& & & \\
MI (\%) & 3.26 & 7.78 & 0.18 \\
Stroke (\%) & 1.09 & 0 & 1.00 \\
Death (\%) & 4.35 & 4.44 & 1.00 \\
CV hospitalisation (\%) & 19.86 & 25 & 0.31 \\
Revascularisation (\%) & 69.56 & 60 & 0.8 \\
Injection site reactions & 15.22 & 15.56 & 0.95 \\
(\%) & & & \\
Other SAE (\%) & 47.83 & 44.44 & 0.65 \\
\hline
\end{tabular}

Discussion NSTEMI ACS treated with all the current evidencedbased therapies still has significant recurrent events. MRC ILAHEART is the first study to evaluate the effects of the anti-inflammatory IL-1ra in ACS. The data indicates that despite encouraging pre-clinical evidence, the inflammatory driver for NSTEMI-ACS is not IL-1 mediated.

\section{ACUTE STENT THROMBOSIS RESULTING IN ST ELEVATION MYOCARDIAL INFARCTION (STEMI) IS ASSOCIATED WITH WORSE CLINICAL OUTCOMES THAN STEMI DUE TO NATIVE CORONARY THROMBOSIS}

doi:10.1136/heartjnl-2011-300198.16

E C Sammut, A Graham, D A Jones, K Rathod, S May, A Jain, S Mohiddin, C Knight, A Mathur, A Wragg. The London Chest Hospital, London, UK

Background Stent thrombosis (ST) is a recognised cause of ST Elevation Myocardial infarction (STEMI) in patients with previous 
percutaneous coronary interventions (PCI). The incidence is increasing and to date outcomes are not well characterised, though there is a suggestion that there is a worse clinical outcome. We therefore sought to compare STEMI caused by ST vs de novo coronary thrombosis to evaluate procedural risk and clinical outcome. Methods Clinical information was analysed from a prospective database on 2421 patients who underwent Primary PCI following STEMI between October 2003 and May 2010 at a London centre. Information was entered at the time of procedure, diagnosis of stent thrombosis made at the time by primary operator and outcome assessed by all-cause mortality information provided by the Office of National Statistics via the BCIS CCAD national audit.

Results Stent thrombosis (ST) accounted for 7.4\% (180/2421) of all STEMIs with a frequency that has increased over time $(5.4 \%$ in 2005 to $9.8 \% 2009)$. ST occurred early (0-30 days) in $36 \%(65 / 180)$, late (30 days -1 year) in 22\% (40/180) and very late (>1 year) in 42\% (75/ $180)$ of pts. Drug-eluting stents (DES) accounted for $48 \%$ of SToverall and $70 \%$ over the past 3 years. Proposed mechanisms included premature discontinuation of anti-platelets (11\%), under-deployment of previous stent insertion (22\%) and underlying prothrombotic conditions (eg, SLE) (6\%). Pts with ST compared to native artery occlusion had higher rates of previous MI $(53.9 \%$ vs $11 \%, \mathrm{p}<0.0001)$ and incidence of multi-vessel disease $(59.8 \%$ vs $51.7 \%, p=0.04$ There was no difference in age, diabetes or cardiogenic shock. See Abstract 16 table 1. Infarct size based on peak enzyme markers was similar ( 2.5 vs $2.2, \mathrm{p}=0.45$ ). In patients with $\mathrm{ST}$, angiographic success (postprocedural Thrombolysis In Myocardial Infarction grade III flow) was worse than in patients with de novo STEMI $(87.2 \%$ vs $93.7 \%, \mathrm{p}=0.02)$. Pts with STEMI due to ST had higher in-hospital MACE ( $11 \%$ vs $3 \%, p=0.0001)$, MACE at 30 days $(19 \%$ vs $6 \%$, $\mathrm{p}<0.0001$ ), this persisted up to 3 years ( $41 \%$ vs $12 \%, p<0.0001)$. See Abstract 16 figure 1. MACE was driven by higher rates of $\mathrm{MI}(7 \%$ vs $2 \%, p<0.0001)$, TVR $(14 \%$ vs $3 \%, p<0.0001)$ and death $(18 \%$ vs $6 \%$, $\mathrm{p}=0.0001$ ). After adjusting for comorbidities, stent thrombosis was an independent predictor of long-term adverse outcome $(\mathrm{OR}=2.1,95 \%$ $\mathrm{CI}=1.3$ to $2.8, \mathrm{p}<0.001)$.

\section{Abstract 16 Table 1}

\begin{tabular}{lccc}
\hline & AST $(\mathbf{n = 1 8 0 )}$ & No AST $(\mathbf{n = 2 2 4 1 )}$ & $\begin{array}{c}\text { Significance } \\
\text { (p value) }\end{array}$ \\
\hline Age & $63.9 \pm 18$ & $62.9 \pm 4$ & 0.406 \\
Multi-vessel disease & $101(59.76 \%)$ & $1011(51.74 \%)$ & 0.045 \\
Previous MI & $96(53.93 \%)$ & $246(10.96 \%)$ & $<0.0001$ \\
Previous CABG & $9(5.06 \%)$ & $60(2.67 \%)$ & 0.020 \\
Diabetes mellitus & $46(25.84 \%)$ & $407(18.14 \%)$ & 0.061 \\
Hypertension & $101(56.74 \%)$ & $975(43.45 \%)$ & 0.002 \\
Hypercholesterolaemia & $108(60.67 \%)$ & $768(34.30 \%)$ & $<0.0001$ \\
Cardiogenic shock & $108(60.67 \%)$ & $768(34.30 \%)$ & $<0.0001$ \\
\hline
\end{tabular}

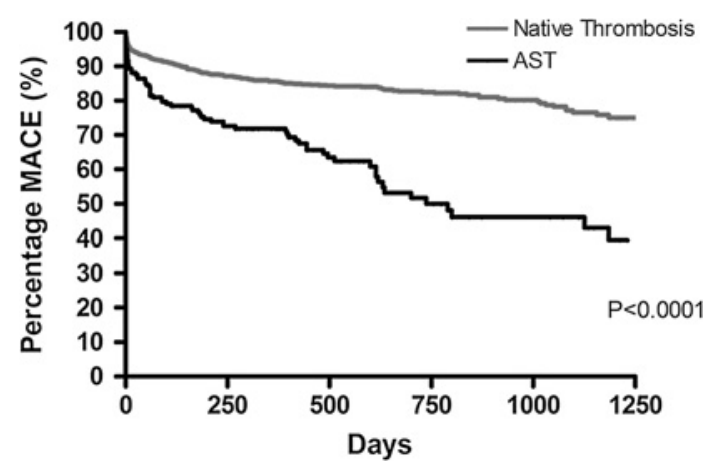

Abstract 16 Figure 1
Conclusion Primary PCI for treatment of ST is less effective, and these patients are at increased risk for in-hospital and long-term mortality compared with patients undergoing primary PCI due to de novo STEMI.

\section{SUDDEN CARDIAC DEATH AND ACUTE MYOCARDIAL INFARCTION: HOW HAS THE PICTURE CHANGED?}

doi:10.1136/heartjnl-2011-300198.17

${ }^{1} \mathrm{G}$ Mole, ${ }^{2} \mathrm{D}$ Watson, ${ }^{3} \mathrm{C}$ Davidson. ${ }^{1}$ Brighton \& Sussex Medical School, Brighton, UK; ${ }^{2}$ Deprtment of Informatics, University of Sussex, Brighton, UK; ${ }^{3}$ Brighton \& Sussex University Hospital, Brighton, UK

Introduction Coronary heart disease (CHD) is a major burden worldwide, particularly in economically developed countries such as the UK. Between 1980 and 2000, deaths from CHD fell by over $50 \%$, and have continued to fall. The cost of CHD manifests itself in mortality, disability and economic impact: this should be looked at in the context of a disease that is preventable.

Methods Data from death certificates and studies with strict clinicopathological criteria on mortality from CHD were accessed. These were analysed in terms of hospital admissions, revascularisation rates and index of multiple deprivation (IMD). Trends in mortality overall and for different age groups were analysed over time to determine how the picture has changed and predict what may happen going into the future. The mortality rates from the catchment area of the Royal Sussex County Hospital and York Hospital were analysed to assess reliability of official figures against strict clinico-pathological inclusion criteria. A range of statistical tests including, linear regression, ANOVA and JoinPoint regression were employed.

Results Between 1993 and 2008 deaths from CHD have fallen by over $50 \%$. The decline has been greater in older age groups particularly the 65-74 age group (the oldest age group analysed). Comparison with data from studies with strict clinico-pathological criteria showed this to be the age group in which official statistics were least accurate. Regression analysis demonstrated that a higher $\mathrm{IMD}$ is associated with increased mortality from CHD $\left(\mathrm{r}^{2}=0.69\right.$, $p<0.001)$. Increased admission rates was not significantly associated with decreased mortality from $\mathrm{CHD}\left(\mathrm{r}^{2}=0.004, \mathrm{p}=0.239\right)$. Increased revascularisation was significantly associated with decreased mortality from CHD $\left(\mathrm{r}^{2}=0.99, \mathrm{p}<0.001\right)$. JoinPoint regression analysis shows a constant rate of decline in mortality from 1993 to 2001 which then decreases faster between 2001 and 2006 before

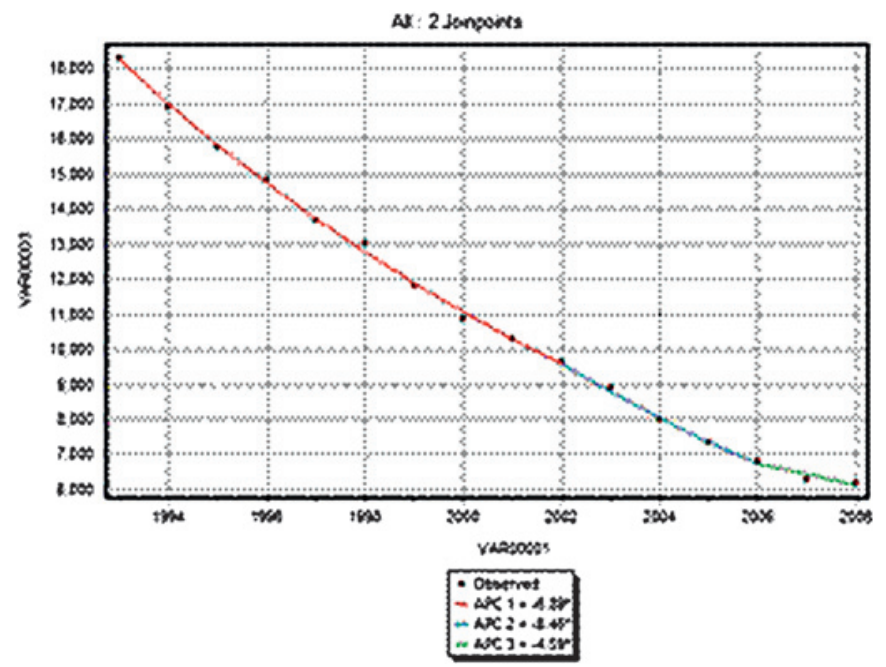

Abstract 17 Figure 1 